\title{
Neuroprotective Effect of Near-Infrared Light in an Animal Model of $\mathrm{Cl}$ Surgery
}

\author{
Ira Strübing ${ }^{a} \quad$ Moritz Gröschel $^{a}$ Susanne Schwitzer ${ }^{a} \quad$ Arne Ernst ${ }^{a}$ \\ Felix Fröhlich $^{a}$ Dan Jiang ${ }^{b}$ Patrick Boyle $^{c}$ Dietmar Basta $^{a}$ \\ aDepartment Otolaryngology at UKB, University of Berlin, Charité Medical School, Berlin, Germany; bepartment \\ Otolaryngology, Guys' and St Thomas' NHS Foundation Trust, London, UK; 'Advanced Bionics Research Center, \\ Hanover, Germany
}

\section{Keywords}

Near-infrared light · Cochlear implant · Residual hearing

\begin{abstract}
Introduction: The preservation of residual hearing has become an important consideration in cochlear implant $(\mathrm{Cl})$ recipients in recent years. It was the aim of the present animal experimental study to investigate the influence of a pretreatment with near-infrared (NIR) light on preservation of sensory hair cells and residual hearing after cochlear implantation. Methods: NIR was applied unilaterally (15 min, 808 $\mathrm{nm}, 120 \mathrm{~mW}$ ) to 8 guinea pigs, immediately before a bilateral scala tympani $\mathrm{Cl}$ electrode insertion was performed. The nonirradiated (contralateral) side served as control. Twentyeight days postoperatively, auditory brainstem responses (ABRs) were registered from both ears to screen for hearing loss. Thereafter, the animals were sacrificed and inner hair cells (IHCs) and outer hair cells (OHCs) were counted and compared between NIR-pretreated and control (contralateral) cochleae. Results: There was no IHC loss upon cochlear implantation. $\mathrm{OHC}$ loss was most prominent on both sides at the apical part of the cochlea. NIR pretreatment led to a statistically significant reduction in OHC loss (by 39.8\%). ABR recordings (across the frequencies $4-32 \mathrm{kHz}$ ) showed a statistically significant difference between the 2 groups and
\end{abstract}

corresponds well with the apical structural damage. Hearing loss was reduced by about $20 \mathrm{~dB}$ on average for the NIRpretreated group ( $p \leq 0.05$ ). Discussion/Conclusion: A single NIR pretreatment in this animal model of $\mathrm{Cl}$ surgery appears to be neuroprotective for residual hearing. This is in line with other studies where several NIR posttreatments have protected cochlear and other neural tissues. NIR pretreatment is an inexpensive, effective, and noninvasive approach that can complement other ways of preserving residual hearing and, hence, should deserve further clinical evaluation in $\mathrm{Cl}$ patients.

(C) 2020 The Author(s) Published by S. Karger AG, Basel

\section{Introduction}

Near-infrared (NIR) light has different effects when applied to mammalian tissue, usually mediated through modulation of the mitochondrial respiratory chain (Fig. 1) and the cytochrome C oxidase in particular [Tsai and Hamblin, 2017]. Its neurotherapeutic efficiency has been widely tested in animal models of Parkinson's [Reinhart et al., 2017], retinal degeneration [Albarracin et al., 2011], in patients following stroke, head trauma or neurodegenerative disorders [Johnstone et al., 2015], and in 


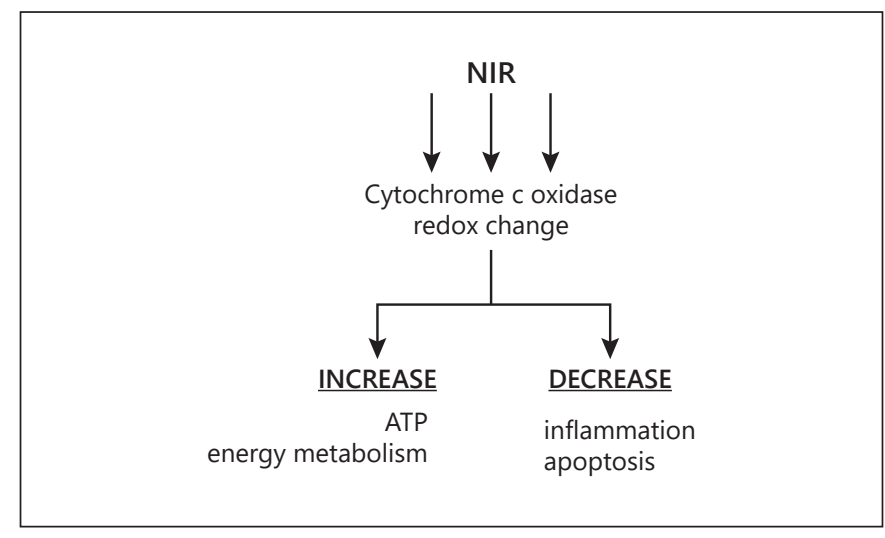

Fig. 1. Effect of NIR light stimulation on the respiratory chain. NIR, near-infrared light.

noise-induced hearing loss [Rhee et al., 2012a]. Animal experiments using NIR to treat noise exposure have shown a decreased cochlear hair cell loss and a significantly lower hearing threshold shift for a daily post-traumatic NIR-treatment over 12 days [Rhee et al., 2012a]. Cell cultures of cochlear outer hair cells (OHCs) showed significantly better recovery from gentamicin exposure if given a daily NIR treatment for 6 days [Rhee et al., 2012b]. Also, a single NIR-preconditioning exposure reduced inflammatory cytokines and markers of oxidative stress upon gentamicin or lipopolysaccharide application [Bartos et al., 2016]. The effects described above are based on photo-biomodulation, that is, a stimulation of mitochondria which in turn increases intracellular ATP production and, thus, reduces apoptosis [Tsai and Hamblin, 2017]. The preservation of residual hearing is becoming more and more important in cochlear implant (CI) surgery as today's CI candidates present with useful amounts of lowfrequency residual hearing. Unfortunately, a significant amount of residual hearing frequently disappears postoperatively, even if the electrode array insertion was performed slowly using soft surgery approaches. The main contributors to hearing loss are the cochlear hair cells which can be mechanically overstimulated by the pressure applied during electrode array insertion [Bas et al., 2012]. Based on the previous findings that an NIR treatment seems to increase the robustness of cochlear hair cells, our aim was to apply this tool in an animal model of CI surgery. Usually, the insertion trauma associated with electrode array insertion in guinea pigs leads to a programmed cell death and a significant loss of the $\mathrm{OHC}$ [Eshraghi et al., 2015]. The NIR would best be applied as a pretreatment, to allow the cochlea to better withstand the surgical approach. The results from a single preconditioning of cochlear hair cells in vitro [Bartos et al., 2016] are very promising for the success of this in vivo histological and functional study. As an additional contribution in the host of international attempts to preserve residual hearing, the noninvasive, inexpensive application of NIR light appears to be both appropriate and interesting.

\section{Materials and Methods}

Eight guinea pigs (Dunkin Hartley, aged 28-30 weeks) were included in this study. Two weeks before surgery, frequency-specific auditory brainstem responses (ABRs) ( $4 \mathrm{~dB}$ increments) were recorded under anesthesia (fentanyl, midazolam, and medetomidine) for the frequencies $4,8,12,16,20,24,28$, and $32 \mathrm{kHz}$ from both ears (for methodology see Basta et al., 2015). Subdermal needle electrodes were placed at the vertex (reference), mastoid (active), and leg (ground). Tone burst stimuli of 3-ms duration and using a Blackman envelope (1.5 ms raise/fall time) were delivered through in-ear high-frequency transducers. Masking of the contralateral ear was achieved using a broadband noise $(2-32 \mathrm{kHz})$ at a level $30 \mathrm{~dB}$ below that of the stimulus intensity. Recording and data acquisition parameters used a 31.1 bursts per second stimulus rate having an alternating phase. There were 1,024 sweeps made per frequency and level. The recording parameters included an amplifier gain of $100 \mathrm{k}$, recording via a bandpass filter of 100-3000 Hz. The sampling rate used to collect electrophysiological data was $32 \mathrm{kHz}$. Stimulation and recording were controlled using the SmartEP software: version 5.33 (Intelligent Hearing Systems, Miami, FL, USA). For each recorded frequency, the peak-to-peak ABR amplitudes, specifically the maximum deflection of waves III/IV were determined for each different stimulus intensity. The hearing threshold was estimated by a stepwise decrease of the stimulus intensity until no electrophysiological response was visually detectable.

Immediately before implantation via the open bulla, each animal had the entire cochlea of 1 randomly selected side pretreated with NIR. The NIR dosage was applied for $15 \mathrm{~min}$, using a wavelength of $808 \mathrm{~nm}$ and a power of $120 \mathrm{~mW}$. Delivery was from a point laser module (Picotronic $\mathrm{GmbH}$, Koblenz, Germany), that, from its output lens that produced a 7-mm diameter beam of collimated NIR. The contralateral side was not pretreated and, hence, served as a control. The laser beam was directed toward the cochlea (Fig. 2), its direction being controlled through a web camera having sufficient bandwidth to image the NIR.

Following NIR pretreatment, all 8 animals were implanted on both sides under anesthesia (fentanyl, midazolam, and medetomidine) with a conically shaped CI electrode array designed for the guinea pig cochlea (Advanced Bionics $\mathrm{GmbH}$, Hanover, Germany). The electrode array diameter was tapered, ranging from 0.4 $\mathrm{mm}$ at the tip to $0.6 \mathrm{~mm}$ at the proximal end. Insertion was performed using a speed of $1 \mathrm{~mm} / \mathrm{s}$, for an insertion depth of $6 \mathrm{~mm}$. After the insertion was complete, the area around the cochlea was closed using muscle tissue, secured using fibrin glue.

Four weeks after surgery, the same frequency-specific ABR recordings described above were repeated. Mean hearing threshold shift was calculated for each individual ear from these results, and 


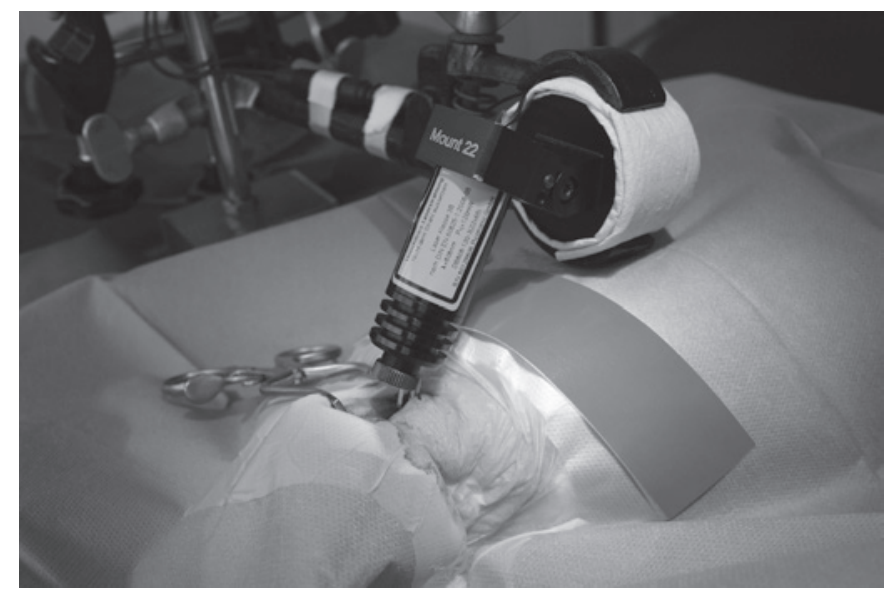

Fig. 2. Animal experimental setup with NIR light irradiation. NIR, near-infrared light.

the results obtained 2 weeks before surgery, with results presented as the mean relative hearing loss.

After the postoperative ABR measurement, the animals were sacrificed, being fixated using a perfusion with $4 \%$ paraformaldehyde delivered via the left heart chamber. The cochleae were carefully taken out. After decalcification overnight (replaced after $8 \mathrm{~h}$ ) with 0.4 M EDTA ( $\mathrm{pH} 8.0$; Roth, Germany), the cochleae were stained by using the Alexa fluor-488-phalloidin staining to visualize F-actin compartments in the cytoskeleton as follows. Cochleae were washed in PBS (Gaithersburg, MD, Gibco, USA) and incubated for 30 min with $0.2 \%$ Triton-X (Sigma-Aldrich, Darmstadt, Germany) in PBS. Afterward the tissue was incubated for $60 \mathrm{~min}$ with Alexa Fluor-488-phalloidin (Molecular Probes, Eugene, OR, USA) solution (1:40 with PBS) on a shaker in the dark and finally washed with PBS. Each organ of Corti was dissected from the modiolus of the cochlea by a micro-scissor to obtain large fragments of half turns. All cochlear fragments were embedded with RotiMount Fluorocare and diamidino phenylindole (DAPI; Roth, Germany) on microscope slides with coverslips. The stained cochleae were magnified microscopically $(20 \times)$ with fluorescence channels for phalloidin $(470 \mathrm{~nm})$ and DAPI $(365 \mathrm{~nm})$. The images were digitized with the AxioCam ICc1 digital camera (Zeiss, Oberkochen, Germany) using the ZEN 2.3 camera software (Zeiss, Oberkochen, Germany). The guinea pig organ of corti represents a clear architecture of 1 row of inner hair cells (IHCs) and 3 rows of OHCs [Wang et al., 2002]. So, it is usual to count only the missing hair cells, which are clearly detectable by the spaces within a row. Figure 3 is a microphotograph showing IHCs and OHCs within the guinea pig cochlea. The number of missing hair cells was counted manually on the basis of the DAPI staining with permanent comparison of the phalloidin staining. The basilar membrane length was measured using the ImageJ $1.47 \mathrm{~d}$ software (National Institutes of Health, USA).

Data were compared between the 2 ears of each animal, that is, with or without NIR pretreatment using either the Wilcoxon test (for not normally distributed data) or $t$ test for paired samples (for normally distributed data). Data distribution was tested with the Kolmogoroff-Smirnoff test. Data from ABR recordings were com-

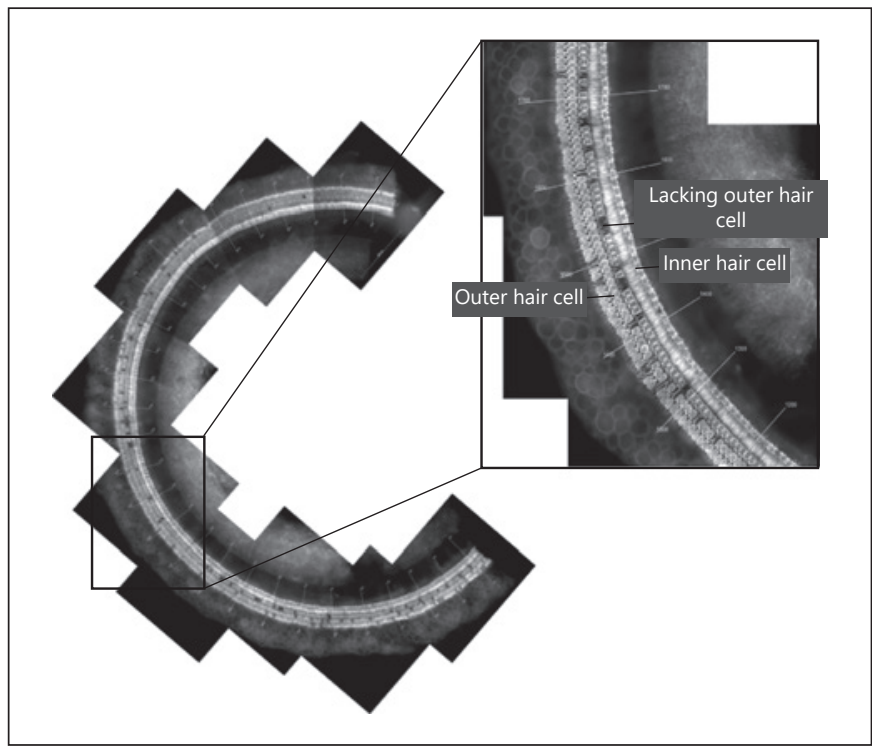

Fig. 3. Whole-mount preparations of the phalloidin-stained guinea pig cochlea.

pared using the $t$ test for paired samples (normally distributed data) with a Bonferoni alpha-correction for multiple comparisons. The total number of missing OHCs following cochlear implantation was compared between sides (pretreated/not pretreated) by the $t$ test for paired samples (normally distributed data). The SPSS software (IBM SPSS Statistics Version 25, IBM Corp., New York, NY, USA) was used for all statistical analyses. The level of significance for all statistical tests was set at $p<0.05$.

As an additional control, temperature measurements of the cochlea were performed during NIR exposure in 2 additional animals (4 ears). The surgery was very similar to that used for the 16 electrode array implantations described above. A thermistor probe $(\mathrm{Pt}$ 100 type $\mathrm{K}$, diameter $0.5 \mathrm{~mm}$ ) was inserted into the cochleostomy. Temperature was measured with a calibrated high-resolution temperature measurement system (T955, Dostmann Electronic $\mathrm{GmbH}$, Wertheim, Germany) before NIR exposure and then after 5,10 , and 15 min of NIR light application.

\section{Results}

\section{Frequency Specific ABR Recordings}

The CI electrode array insertion induced the highest amount of hearing loss in the low-frequency range and the lowest amount of hearing loss in the high-frequency range (Fig. 4). There was a statistically significant difference between the pretreated ears and the control ears. The level of significance was reached for frequencies of $8 \mathrm{kHz}$ and above (Fig. 4). The average hearing loss and standard deviations of the control and pretreated ears were $33.5 \pm$ $5.1 \mathrm{~dB}$ and $18.4 \pm 5.8 \mathrm{~dB}$, respectively. The protective ef- 
Fig. 4. Threshold shift of ABR following cochlear implantation in pretreated (NIR light) ears and controls. Error bars represent standard error. Asterisks indicate significant differences between the groups $(p<0.05)$. ABR, auditory brainstem response; NIR, near-infrared light.

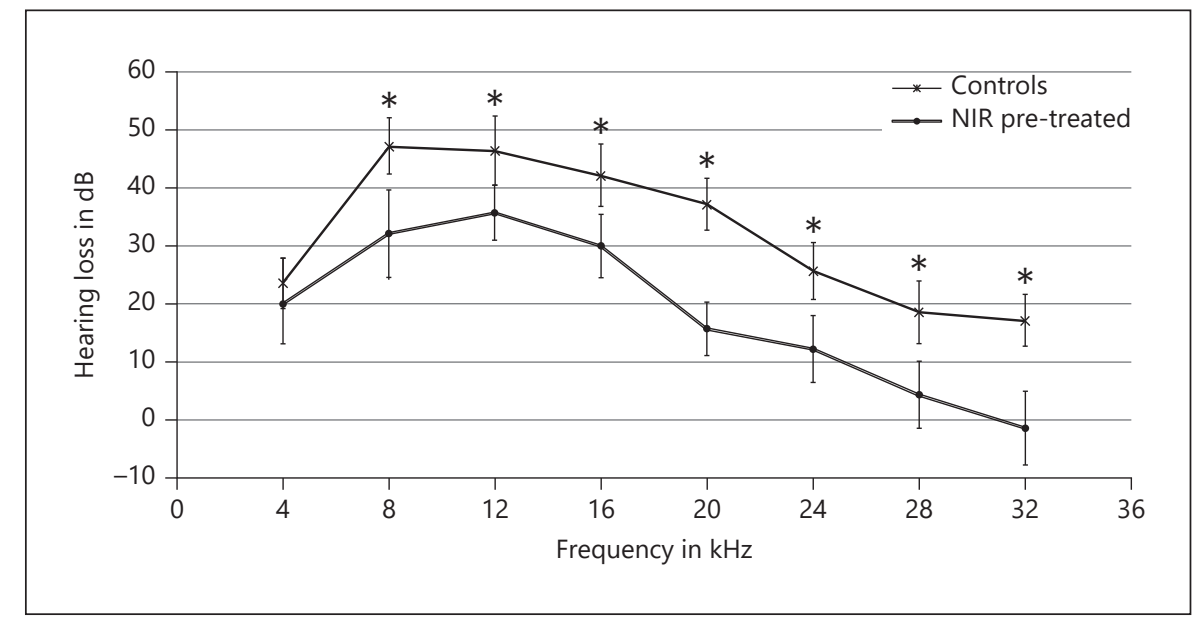

fect of the NIR pretreatment amounted to between 10.7 and $21.4 \mathrm{~dB}$ depending on the individual frequency being compared.

\section{Temperature Measurements}

The average local intra-cochlear temperature increase after $15 \mathrm{~min}$ of NIR pretreatment was $0.95 \pm 0.12^{\circ} \mathrm{C}$. The increase from the baseline $\left(34.3 \pm 0.15^{\circ} \mathrm{C}\right)$ was nearly linear, with values of $34.6 \pm 0.11^{\circ} \mathrm{C}, 34.8 \pm 0.06^{\circ} \mathrm{C}$, and 35.25 $\pm 0.2^{\circ} \mathrm{C}$ after 5,10 , or $15 \mathrm{~min}$, respectively.

\section{Hair Cell Counts}

When analyzing the cochleae after sacrifice, no loss of IHCs was detected bilaterally. Cochlear implantation induced a higher OHC loss in the apical part of the cochlea than the basal part (Fig. 5). All parts of the cochlea were similarly affected by the NIR pretreatment in that the differences in $\mathrm{OHC}$ counts between pretreated cochleae and control cochleae were uniformly distributed along the cochleae.

The OHC counts showed statistically significant differences between the pretreated and the control ears ( $p=$ 0.006; Fig. 3). This results in a reduction of $\mathrm{OHC}$ loss by $39.8 \%$ for the pretreated group. Along the whole length of the basilar membrane, the controls showed an average OHC loss of $129.3 \pm 7.8$ cells, in contrast to $88.7 \pm 7.3$ cells in the pretreated group (Fig. 6).

\section{Discussion}

Our results confirm the neuroprotective effect of NIR in our animal model of CI surgery. As demonstrated earlier, NIR can penetrate the bony shell of the skull or - in our case - the temporal bone and in particular the cochlea [Jagdeo et al., 2012]. This ability makes NIR with an 808$\mathrm{nm}$ wavelength an interesting tool for sensory organs (retina and cochlea), and also for application in the brain. Apart from those acute lesions as induced with a CI electrode array, chronic neurodegenerative disorders (e.g., Parkinson's [Reinhart et al., 2017]) respond to this type of irradiation in animal models and human applications as well.

Our findings demonstrated neuroprotective effects not only audiological by ABR recordings but also by microstructural analysis of the cochlear OHCs. Interestingly, the preservation of residual hearing by NIR could be much higher than we observed. The intra-individual comparison of the pretreated and control sides might have masked the so-called remote effects of NIR treatment [Ganeshan et al., 2019]. This new concept of a systemic NIR action shows a protective effect on neuronal tissue independent of the place of NIR treatment. Even if the effect on the area directly exposed to NIR is significantly higher than elsewhere, the remaining tissue of the same animal may still be influenced.

Surprisingly, the apical OHCs were more affected than the basal ones, where the electrode array was located and some insertion trauma might have been expected [Roland and Wright, 2006]. This observation corresponds well with the higher hearing loss recorded in the low-frequency region. It appears that intracochlear pressure changes applied to the apical part of the cochlea during the insertion process influence $\mathrm{OHC}$ loss more than the electrode array touching cochlear structures in the basal turn [Robertson and Johnstone, 1979; Todt et al., 2014]. These pressure changes were minimized as far as possible since the electrode was inserted very slowly and the arm of the surgeon was supported
98

Audiol Neurotol 2021;26:95-10 DOI: $10.1159 / 000508619$
Strübing/Gröschel/Schwitzer/Ernst/ Fröhlich/Jiang/Boyle/Basta 
Fig. 5. OHC distribution along the basilar membrane (basal to apical) of controls (a) and NIR light-pretreated ears (b) following cochlear implantation. Error bars represent standard error. OHC, outer hair cell; NIR, near-infrared light.

Fig. 6. Total number of missing OHCs following cochlear implantation in pretreated (NIR light) ears and controls. Asterisks indicate highly significant differences between the groups $(p<0.01)$. Error bars represent standard error. OHC, outer hair cell; NIR, near-infrared light.
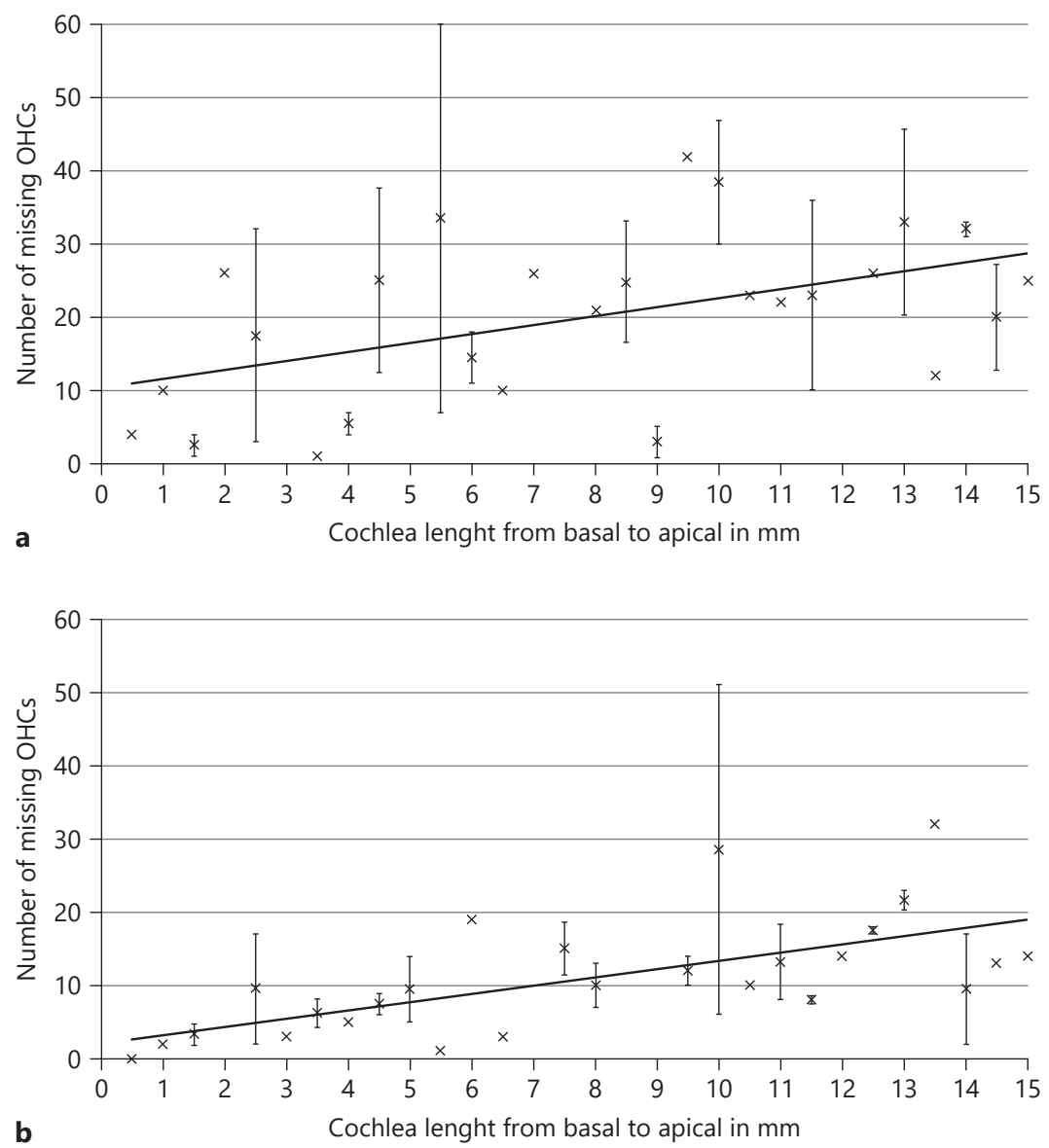

b

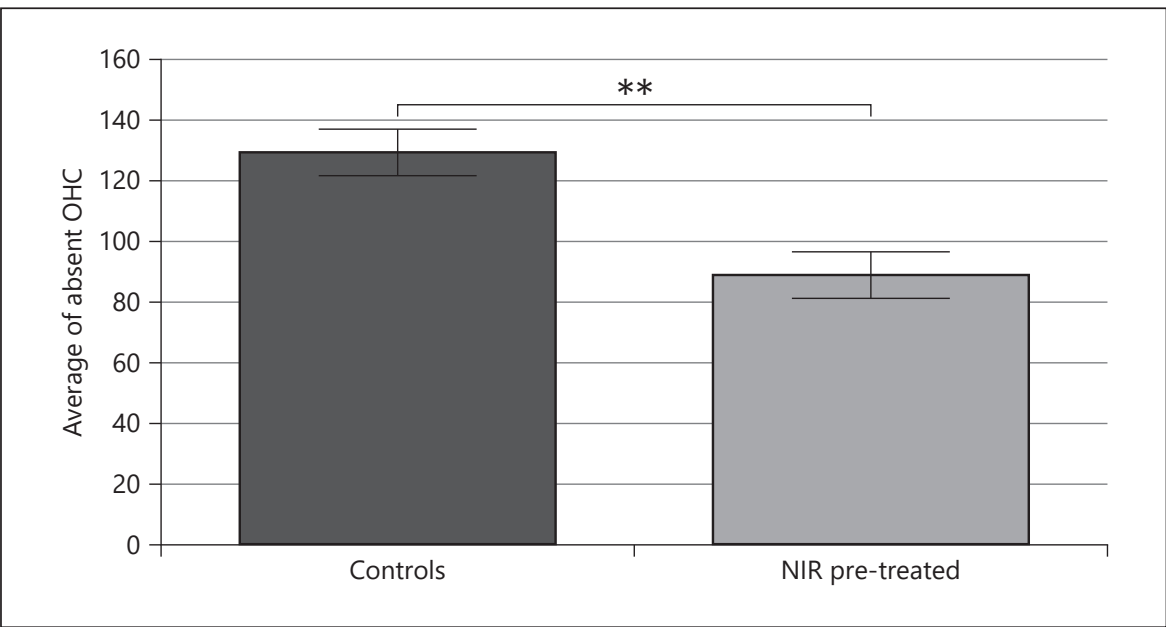

during insertion [Todt et al., 2016]. It is possible this is why the IHCs were all perfectly preserved. In addition, the insertion of the electrode array could possibly induce neuroprotective mechanisms, responding to the mechanical injury.
Such effects have been previously described in studies of retinal injury [Faktorovich et al., 1992]. Thus, the region where the electrode array was located could be more protected from neurodegeneration than apical regions which 
receive pressure changes only. Even if the threshold shift of $\mathrm{ABR}$ responses corresponds well with the region of highest OHC loss, the insertion-induced threshold shift could not be explained alone by approximately 130 missing $\mathrm{OHC}$ out of almost 6,300 in the guinea pig cochlea. The same holds true for the protective effect. It is very unlikely that the rescue of approximately $40 \mathrm{OHCs}$ can explain protection of 20 $\mathrm{dB}$ on average for the hearing threshold. Besides the effects observed for cochlear OHCs, there must be other pathological mechanisms arising from electrode array insertion (e.g., lateral wall pathologies and synaptic changes [Wright and Roland, 2013; Reiss et al., 2015]). Fortunately, the NIR pretreatment seems to also have a protective effect on these uncovered mechanisms. Future research should investigate these additional processes in more detail.

Surprisingly, a single short NIR pretreatment was similarly effective in our CI experiments to several longer posttreatments (60 min per day over 12 days), particularly considering a comparable wavelength and power [Rhee et al., 2012a]. This could be related to different pathologies induced by noise and our mechanical intervention. Possibly, the efficacy of a pretreatment is higher due to protective mechanisms being activated before any structural damage is induced. It could be more complicated to reverse structural changes (e.g., apoptotic mechanisms) than to prevent them occurring in the first place. More research is also necessary to clarify this hypothesis.

Several groups have proposed various steps to preserve residual hearing following cochlear implantation (e.g., drug application, surgical technique modifications, and electrode design variations). However, the long-term results are not yet very promising [Snels et al., 2019].

NIR light with its intracellular effects on the mitochondrial respiratory chain increases the proton transport out of mitochondria and, thus, increases the production of ATP, so that inflammatory and apoptotic mechanisms accompanying any neurotrauma (e.g., insertion of a CI electrode) are downregulated [Tsai and Hamblin, 2017]. As a result, this rather inexpensive, noninvasive, one-time (before surgery) NIR light application appears to be an another promising method in the set of tools available to help preserve residual hearing.

\section{Conclusion}

A single NIR pretreatment in this animal model of CI surgery appears equally neuroprotective to several previously demonstrated posttreatments, in cochlear and in other neural tissues. NIR pretreatment is an inexpensive, effective, noninvasive approach that can be combined with other approaches to help preserve residual hearing and, hence, deserves further clinical application and evaluation in CI patients.

\section{Statement of Ethics}

The Board for Granting Permission to Perform Animal Experiments (Land Berlin) approved the study (approval number: $G$ $0146 / 14)$.

\section{Conflict of Interest Statement}

A.E. is a member of the Surgical Advisory Board (Europe) for Advanced Bionics GmbH. P.B. is employed in a scientific role by Advanced Bionics GmbH, Hanover, Germany. All other authors disclose no conflicts of interest.

\section{Funding Sources}

This study was supported by Advanced Bionics GmbH, Hanover, Germany.

\section{Author Contributions}

Ira Strübing - acquisition, analysis of data, drafting the work, and final approval of the version to be published. Moritz Gröschel - acquisition, analysis of data and interpretation, and final approval of the version to be published. Arne Ernst - conception of the work, revising it critically for important intellectual content, and final approval of the version to be published. Susanne Schwitzer - acquisition, revising it critically for important intellectual content, and final approval of the version to be published. Felix Fröhlich - revising it critically for important intellectual content and final approval of the version to be published. Dan Jian - conception of the work, revising it critically for important intellectual content, and final approval of the version to be published. Patrick Boyle - conception of the work, revising it critically for important intellectual content, and final approval of the version to be published. Dietmar Basta - conception of the work, analysis of data, drafting the work, revising it critically for important intellectual content, and final approval of the version to be published. All authors were accountable for all aspects of the work in ensuring that questions related to the accuracy or integrity of any part of the work are appropriately investigated and resolved. 


\section{References}

Albarracin R, Eells J, Valter K. Photobiomodulation protects the retina from light-induced photoreceptor degeneration. Invest Ophthalmol Vis Sci. 2011 Jun 1;52(6):3582-92.

Bas E, Dinh CT, Garnham C, Polak M, Van de Water TR. Conservation of hearing and protection of hair cells in cochlear implant patients with residual hearing. Anat Rec. 2012 Nov;295(11):1909-27.

Bartos A, Grondin Y, Bortoni ME, Ghelfi E, Sepulveda R, Carroll J, et al. Pre-conditioning with near infrared photobiomodulation reduces inflammatory cytokines and markers of oxidative stress in cochlear hair cells. J Biophotonics. 2016 Dec;9(11-12):1125-35.

Basta D, Götze R, Gröschel M, Jansen S, Janke O, Tzschentke B, et al. Bilateral changes of spontaneous activity within the central auditory pathway upon chronic unilateral intracochlear electrical stimulation. Otol Neurotol. 2015 Dec;36(10):1759-65.

Eshraghi AA, Lang DM, Roell J, Van De Water TR, Garnham C, Rodrigues H, et al. Mechanisms of programmed cell death signaling in hair cells and support cells post-electrode insertion trauma. Acta Otolaryngol. 2015 Apr; 135(4):328-34.

Faktorovich EG, Steinberg RH, Yasumura D, Matthes MT, LaVail MM. Basic fibroblast growth factor and local injury protect photoreceptors from light damage in the rat. J Neurosci. 1992 Sep;12(9):3554-67.
Ganeshan V, Skladnev NV, Kim JY, Mitrofanis J, Stone J, Johnstone DM. Pre-conditioning with remote photobiomodulation modulates the brain transcriptome and protects against MPTP insult in mice. Neuroscience. 2019 Feb;400:85-97.

Jagdeo JR, Adams LE, Brody NI, Siegel DM. Transcranial red and near infrared light transmission in a cadaveric model. PLoS One. 2012;7(10):e47460

Johnstone DM, Mitrofanis J, Stone J. Targeting the body to protect the brain: inducing neuroprotection with remotely-applied near infrared light. Neural Regen Res. 2015 Mar; 10(3):349-51

Reinhart F, Massri NE, Torres N, Chabrol C, Molet J, Johnstone DM, et al. The behavioural and neuroprotective outcomes when $670 \mathrm{~nm}$ and $810 \mathrm{~nm}$ near infrared light are applied together in MPTP-treated mice. Neurosci Res. 2017 Apr;117:42-7.

Reiss LA, Stark G, Nguyen-Huynh AT, Spear KA, Zhang H, Tanaka C, et al. Morphological correlates of hearing loss after cochlear implantation and electro-acoustic stimulation in a hearing-impaired guinea pig model. Hear Res. 2015 Sep;327:163-74.

Rhee CK, Bahk CW, Kim SH, Ahn JC, Jung JY, Chung PS, et al. Effect of low-level laser treatment on cochlea hair-cell recovery after acute acoustic trauma. J Biomed Opt. 2012a Jun; 17(6):068002.

Rhee CK, He P, Jung JY, Ahn JC, Chung PS, Suh MW. Effect of low-level laser therapy on cochlear hair cell recovery after gentamicin-induced ototoxicity. Lasers Med Sci. 2012b Sep; 27(5):987-92.
Robertson D, Johnstone BM. Aberrant tonotopic organization in the inner ear damaged by kanamycin. J Acoust Soc Am. 1979 Aug;66(2): 466-9.

Roland PS, Wright CG. Surgical aspects of cochlear implantation: mechanisms of insertional trauma. Adv Otorhinolaryngol. 2006;64:1130.

Snels C, IntHout J, Mylanus E, Huinck W, Dhooge I. Hearing preservation in cochlear implant surgery: a meta-analysis. Otol Neurotol. 2019 Feb;40(2):145-53.

Todt I, Ernst A, Mittmann P. Effects of different insertion techniques of a cochlear implant electrode on the intracochlear pressure. Audiol Neurootol. 2016;21(1):30-7.

Todt I, Mittmann P, Ernst A. Intracochlear fluid pressure changes related to the insertional speed of a CI electrode. Biomed Res Int. 2014; 2014:507241.

Tsai SR, Hamblin MR. Biological effects and medical applications of infrared radiation. J Photochem Photobiol B. 2017 May;170:197-207.

Wang J, Dib M, Lenoir M, Vago P, Eybalin M, Hameg A, et al. Riluzole rescues cochlear sensory cells from acoustic trauma in the guineapig. Neuroscience. 2002;111:635-48.

Wright CG, Roland PS. Vascular trauma during cochlear implantation: a contributor to residual hearing loss? Otol Neurotol. 2013 Apr; 34(3):402-7. 